\title{
Some Thoughts on Precision Medicine
}

\section{Shein-Chung Chow ${ }^{1 *}$ and Fuyu Song ${ }^{2}$}

${ }^{1}$ Duke University School of Medicine, Durham, North Carolina, USA

${ }^{2}$ Peking University Clinical Research Institute, Peking University Health Science Center, Beijing, China

\section{Introduction}

In clinical trials, a typical approach for evaluation of safety and efficacy of a test treatment under investigation is to first test for the null hypothesis of no treatment difference in efficacy based on clinical data collected under a valid trial design. If significant, the investigator would reject the null hypothesis of no treatment difference and then conclude the alternative hypothesis that there is a difference in favor of the test treatment. If there is a sufficient power for correctly detecting a clinically meaningful difference (improvement) when such a difference truly exists, we claim that the test treatment is efficacious. The test treatment will then be reviewed and approved by the regulatory agency such as the United States Food and Drug Administration (FDA) if the test treatment is well tolerated and there appears to be no safety concerns. We will refer to medicine developed based on this typical approach as traditional medicine.

In his State of the Union address early this year, President Obama announced that he's launching the Precision Medicine Initiative - a bold new research effort to revolutionize how we improve health and treat disease. As indicated by President Obama, precision medicine is an innovative approach that takes into account individual differences in people's genes, environments, and lifestyles. Unlike traditional approach (traditional medicine), most medical treatments have been designed for the average patient. This one-size-fits-all approach, treatments can be very successful for some patients but not for others. Precision medicine, on the other hand, gives medical professionals the resources they need to target the specific treatments of the illnesses we encounter [1]. In response to President Obama's Precision Medicine Initiative, National Institutes of Health (NIH) kicked off cohort grants for precision medicine to develop treatments tailored to an individual based on their genetics and other personal characteristics subsequently [2]. Seeking for precision medicine of cure has become the center of clinical research in pharmaceutical development since then.

\section{Precision Medicine}

Unlike traditional medicine, precision medicine (PM) is referred to as a medical model that proposes the customization of healthcare, with medical decisions, practices, and/or products being tailored to the individual patient [3]. In this model, diagnostic testing is often employed for selecting appropriate and optimal therapies based on the context of a patient's genetic content or other molecular or cellular analysis. Tools employed in PM could include molecular diagnostics, imaging, and analytics/software. This has led to biomarker development in genomics studies for target clinical trial. A validated biomarker (diagnostic tool) is then used to identify patients who are most likely to respond to the test treatment under investigation in the enrichment process of the target clinical trials [4-7]. As a result, precision medicine will benefit subgroup of patients who are biomarker positive. In practice, however, there may exist no perfect diagnostic tool for determining whether a given patient is with or without molecular target in the enrichment process of the target clinical trials. Possible misclassification, which could cause significant bias in assessment of treatment effect in target clinical trials, is probably the most challenging issue in precision medicine.
As an example, let $\mathrm{Y}_{\mathrm{ij}}$ be the responses of the jth subject in the ith group, where $\mathrm{j}=1, \ldots, \mathrm{n}_{\mathrm{i}} ; \mathrm{I}=\mathrm{T}, \mathrm{C} . \mathrm{Y}_{\mathrm{ij}}$ are assumed approximately normality distributed with homogeneous variances between the test and control treatments. Also, let $\mu_{\mathrm{T}+}, \mu_{\mathrm{C}+}\left(\mu_{\mathrm{T}-}, \mu_{\mathrm{C}_{-}}\right)$be the means of test and control groups for the patients with (without) the molecular target. Table 1 summarizes population means by treatment and diagnosis.

In target clinical trials, it is of interest to estimate the treatment effect for the patients truly having the molecular target, i.e., $\theta=\mu_{\mathrm{T}+}-\mu_{\mathrm{C}+}$. However, this effect may be contaminated due to misclassification, i.e., for those subjects who do not have the molecular target but got positive diagnosed results and those subject who have the molecular target but got negative diagnosed results. The following hypothesis for detecting a clinically meaningful treatment difference in the patient population truly with the molecular target is of interest:

$$
\mathrm{H}_{0}: \mu_{\mathrm{T}+}-\mu_{\mathrm{C}+}=0 \quad \text { vs. } \quad \mathrm{H}_{\mathrm{a}}: \mu_{\mathrm{T}+}-\mu_{\mathrm{C}+} \neq 0
$$

Let $\overline{\mathrm{y}}_{\mathrm{T}}$ and $\overline{\mathrm{y}}_{\mathrm{C}}$ be the sample means of test and control treatments, respectively. Since no diagnostic test is perfect for diagnosis of the molecular target of interest without error, therefore, some patients with a positive diagnostic result may in fact do not have the molecular target. It follows that

$$
\mathrm{E}\left(\overline{\mathrm{y}}_{\mathrm{T}}-\overline{\mathrm{y}}_{\mathrm{C}}\right)=\gamma\left(\mu_{\mathrm{T}+}-\mu_{\mathrm{C}+}\right)+(1-\gamma)\left(\mu_{\mathrm{T}-}-\mu_{\mathrm{C}-}\right),
$$

where $\gamma$ is the positive predicted value, which is often unknown. Thus, an accurate and reliable estimate of $\gamma$ is the key to the success of target clinical trials [7] and hence precision medicine.

\section{Precision Medicine versus Personalized Medicine}

The term precision medicine is often mixed up with the term personalized medicine. To distinguish the difference between precision medicine and personalized (or individualized) medicine, the National

\begin{tabular}{|c|c|c|c|c|c|}
\hline $\begin{array}{c}\text { Positive } \\
\text { diagnosis }\end{array}$ & $\begin{array}{c}\text { True target } \\
\text { condition }\end{array}$ & $\begin{array}{c}\text { Indicator of } \\
\text { diagnostic }\end{array}$ & Test group & $\begin{array}{c}\text { Control } \\
\text { group }\end{array}$ & Difference \\
\hline+ & + & $\gamma$ & $\mu_{\mathrm{T}+}$ & $\mu_{\mathrm{C}+}$ & $\mu_{\mathrm{T}+}-\mu_{\mathrm{C}+}$ \\
\hline & - & $1-\gamma$ & $\mu_{\mathrm{T}-}$ & $\mu_{\mathrm{C}-}$ & $\mu_{\mathrm{T}-}-\mu_{\mathrm{C}-}$ \\
\hline
\end{tabular}

Note that $\gamma$ is the positive predicted value

Table 1: Population means by treatment and diagnosis.

*Corresponding author: Shein-Chung Chow, Professor, Biostatistics and Bioinformatics, Duke University School of Medicine, 2424 Erwin Road, Hock Suite 1102, Room 11068, Durham, North Carolina, USA, Tel: (919) 668-7523; E-mail: sheinchung.chow@duke.edu

Received December 17, 2015; Accepted December 18, 2015; Published December 26, 2015

Citation: Chow SC, Song F (2015) Some Thoughts on Precision Medicine. J Biom Biostat 6: 269. doi:10.4172/2155-6180.1000269

Copyright: $\odot 2015$ Chow SC, et al. This is an open-access article distributed unde the terms of the Creative Commons Attribution License, which permits unrestricted use, distribution, and reproduction in any medium, provided the original author and source are credited. 


\begin{tabular}{|c|c|c|c|}
\hline Characteristic & Traditional Medicine & Precision Medicine & Personalized Medicine ${ }^{*}$ \\
\hline Active Ingredient & Single & Single & Multiple \\
\hline Target population & Population & Population & Individuals \\
\hline Primary focus & Mean & Inter-subject variability & Intra-subject variability \\
\hline Dose/Regimen & Fixed & Fixed & Flexible \\
\hline Beneficial & Average patient & Subgroup of patients & Individual patients \\
\hline Statistical Method & $\begin{array}{l}\text { Hypotheses testing } \\
\text { Confidence interval }\end{array}$ & $\begin{array}{l}\text { Hypotheses testing } \\
\text { Confidence interval }\end{array}$ & $\begin{array}{l}\text { Hypotheses testing } \\
\text { Confidence interval }\end{array}$ \\
\hline Use of biomarker & No & Yes & Yes \\
\hline Blinding & Yes & Yes & May be difficult \\
\hline Objective & Accuracy & Accuracy Precision & $\begin{array}{c}\text { Accuracy } \\
\text { Precision } \\
\text { Reproducibility }\end{array}$ \\
\hline Study design & Parallel/crossover & $\begin{array}{l}\text { Parallel/crossover } \\
\text { Adaptive design }\end{array}$ & $\begin{array}{l}\text { Parallel/Crossover } \\
\text { Adaptive design }\end{array}$ \\
\hline Probability of success & Low & Mild-to-moderate & High \\
\hline
\end{tabular}

*Personalized medicine = Individualized medicine

Table 2: Precision medicine versus personalized medicine.

Research Council (NRC) indicates that precision medicine refers to the tailoring of medical treatment to the individual characteristics of each patient. It does not literally mean the creation of drugs or medical devices that are unique to a patient, but rather the ability to classify individuals into subpopulations that differ in their susceptibility to a particular disease, in the biology and/or prognosis of those diseases they may develop, or in their response to a specific treatment. In summary, precision medicine is to benefit subgroup of patients with the diseases under study, while personalized medicine is to benefit individual subjects with the diseases under investigation.

Statistically, the term precision is usually referred to the degree of closeness of the observed data to the truth. High degree of closeness is an indication of high precision. Thus, precision is related to the variability associated with the observed data. In practice, the variability associated with observed data includes (1) intra-subject variability, (2) inter-subject variability, and (3) variability due to subject-bytreatment interaction. As a result, precision medicine can be viewed as the identification of subgroup population with larger effect size (i.e., smaller variability) assuming that the difference in mean response is fixed. Consequently, precision medicine focuses on minimizing intersubject variability, while personalized medicine focuses on minimizing intra-subject variability. Table 2 provides a comparison between precision medicine and personalized medicine.

\section{Future Perspectives}

As indicated earlier, traditional medicine can only benefit average patients with the diseases under study, while precision medicine can further benefit a subgroup of patients who have certain characteristics (e.g., molecular target). On the other hand, the ultimate goal of personalized (or individualized) medicine is seeking cure for individual patients if it is not impossible. President Obama's Precision Medicine Initiative is an important step moving away from the traditional medicine and toward to personalized (or individualized) medicine so that individual patients could be beneficial. For this purpose, traditional
Chinese medicine, which often consists of multiple components and focuses on global dynamic harmony (or balance) within individual patients, is expected to be the center of personalized medicine development moving toward the next century [8]. For achieving this ultimate goal, regulatory requirement and quantitative/statistical methods for assessment of treatment effect for drug products with multiple components are necessarily developed.

\section{References}

1. News Release (2015) FACT SHEET: President Obama's Precision Medicine Initiative. The White House, Office of the Press Secretary, USA.

2. McCarthy J (2015) NIH kicks off cohort grants for precision medicine USA

3. NRC (2011) Toward Precision Medicine: Building a Knowledge Network for Biomedical Research and a New Taxonomy of Disease. Committee on A Framework for Developing a New Taxonomy of Disease; National Research Council. The National Academies Press, Washington DC, USA.

4. FDA (2005) The draft concept paper on drug-diagnostic co-development. The US Food and Drug Administration, Rockville, MD.

5. FDA (2007a) Guidance on pharmacogenetic tests and genetic tests for heritable marker. The US Food and Drug Administration Rockville, MD.

6. FDA (2007b) Draft guidance on in vitro diagnostic multivariate index assays. The US Food and Drug Administration Rockville, MD.

7. Liu JP, Lin JR, Chow SC (2009) Inference on treatment effects for targeted clinical trials under enrichment design. Pharmaceutical Statistics 8: 356-370.

8. Chow SC (2015) Quantitative Methods for Traditional Chinese Medicine Development. Chapman and Hall/CRC Press, Taylor and Francis, New York. 